\title{
V2X-Based Event Acquisition and Reproduction Architecture with Event-Sourcing
}

\author{
Sangkon Han \\ Department of Computer Science Engineering, \\ Pusan National University \\ 2, Busandaehak-ro 63beon-gil, Geumjeong-gu, \\ Busan, Republic of Korea \\ 82-10-6783-8319 \\ sangkon@pusan.ac.kr
}

\author{
Jung-in Choi \\ Software Education Center, \\ Pusan National University \\ 2, Busandaehak-ro 63beon-gil, Geumjeong-gu, \\ Busan, Republic of Korea \\ 82-10-7448-7332 \\ jungin.choi@pusan.ac.kr
}

\begin{abstract}
Currently, many levels of autonomous driving are being rapidly developed and are near deployment. No human intervention is required above level 4 of autonomous driving. Imperatively, a method for preventing and reproducing accidents that may occur during autonomous driving is desirable. In this study, the state change that occurs in V2X (vehicle to everything) environment is treated as first-class data to examine the cause by reproducing the accident when an accident occurs. We propose a framework that reproduces and restores state using event sourcing techniques that store first-class data.
\end{abstract}

\section{CCS Concepts}

- Computer systems organization $\rightarrow$ Architectures $\rightarrow$ Other architectures $\rightarrow$ Data flow architectures.

\section{Keywords}

Event-Sourcing; Event Acquisition; Reproduction Architecture;

\section{INTRODUCTION}

Recently, there has been much advancement in research and development involving level 4 autonomous driving, as defined by National Highway Traffic Safety Administration (NHTSA). Autonomous vehicles are classified into levels 0 to 4 according to their degree of automation and technology level of autonomous vehicles [1]. Autonomous driving levels 0 to 2 require a driver to look ahead and monitor the driving environment. Level 3 requires some forward monitoring, while level 4 is fully autonomous and requires no drivers. Accordingly, research on how to prevent and reproduce accidents in autonomous driving is needed. Self-driving in level 4 has no human interventions. It is imperative relative to vehicle safety to reproduce accident situations to examine the cause, and infer preventive measures [2]. In current autonomous driving, surrounding situations are recognized using a front camera and light detection and ranging (LIDAR). In reproducing an event, data is retrieved from a storage mechanism that receives data from the camera and LIDAR.

\footnotetext{
Permission to make digital or hard copies of all or part of this work for personal or classroom use is granted without fee provided that copies are not made or distributed for profit or commercial advantage and that copies bear this notice and the full citation on the first page. Copyrights for components of this work owned by others than ACM must be honored. Abstracting with credit is permitted. To copy otherwise, or republish, to post on servers or to redistribute to lists, requires prior specific permission and/or a fee. Request permissions from Permissions@acm.org.

ICCDE '20, January 4-6, 2020, Sanya, China

(C) 2020 Association for Computing Machinery.

ACM ISBN 978-1-4503-7673-0/20/01 ..\$15.00

https://doi.org/10.1145/3379247.3379290
}

Additionally, following the development of 5G network technology and low power technology, there are diverse devices participating in V2X. 5G networks have low latency and are beneficial for large data transfers. Particularly, communication distance is wider than existing networks, and more devices and large data can be utilized [3]. Therefore, there is the need for a method of storing and utilizing these data. The V2X-event representation architecture proposed in this study presents a method of storing and reproducing an event by handling the change in vehicle and surrounding environment.

\section{RELATED WORK}

\subsection{Event Sourcing}

Event sourcing is a technique that treats and stores actions or data from state changes or data is generated [4]. Event sourcing is suitable for responsive systems [5] using message delivery. Additionally, the scale of the system is uncomplicated owing to the nature of the responsive system. However, developers are familiar with the RDBMS (relational database management system) approach of storing a final state.

Consequently, event sourcing that treats all events as one piece of data, is a strange technique to programmers familiar with the traditional methods $[6,7]$.

Event sourcing treats all sequentially recorded events occurring in the domain model as first-class citizen data. This data is called a "domain event." It is a collection of events from the beginning to the present.

Stored events can be actively used for machine learning. Machine learning relies heavily on available data. Accuracy of predictions increases with available data. As event sourcing involves storing every event in the domain, more data is available to analyze, leading to accurate predictions. For example, in online shopping malls, some customers keep orders in their shopping carts for longer periods than others. Event sourcing allows tracking user behaviors such as add, remove, and create. Machine learning can be used to predict $[8,9]$ the likelihood that customers who pay unreservedly will next purchase a product and send a discount.

Domain events are recorded in "event-store," and restoring all events stored in the event-store restores the current state of domain events. Individual events included in domain events are not modified or deleted because they must be restored using domain events. 


\subsection{Reactive System}

A reactive system is not a specific system. It satisfies the following four characteristics: 1) Responsive, 2) Resilient, 3) Elastic, and 4) Message Driven.

Reactive systems notably respond quickly to provide a user with a positive experience. If a system is responsive, it can provide a consistent quality of service and promote new interactions [5].

To ensure immediate responsiveness, maintaining responsiveness during a system failure, called resilient, is essential. Elasticity is the property of maintaining responsiveness as a system's workload changes.

To construct such flexible systems, a message protocol based on an asynchronous protocol is actively used. The message-based system is a queue distribution system that prevents system overload and operate a stable system.

\section{ADVANTAGES AND DISADVANTAGES OF EVENT SOURCING}

Event sourcing is ideal for distributed systems, allowing easy scale-up and reliable logs. However, its major disadvantage is not suitable for reading. This chapter describes advantages and disadvantages of event sourcing.

\subsection{Advantages of Event Sourcing}

Firstly, event sourcing is easy to scale up because it is suitable for distributed systems. Event sourcing is desirable for responsive systems that use message delivery and does not require modification and deletion operations during event storage. The data structures and operations used in event sourcing are simple. This allows leveraging common repositories such as relational databases, document databases, key-value stores, and file systems. As the event store does not require modification and deletion operations, it applicable to distributed systems even if an existing store is used.

Secondly, a reliable log can be secured. For example, almost any state change that occurs in a V2X environment can be saved as an event. As no modification and deletion operations are required, there is no side-effect during saving an event. Applying the collected data sequentially to a specific time can reproduce and restore the state at that time. Thus, at any time, states can be restored and verified based on reliable data $[10,11]$. Some similar concepts are logging and monitoring. Logging and monitoring functions focus on errors or exceptions that occur in a system or architecture. Event sourcing is a major difference between dealing with actual values and messages.

\subsection{Disadvantages of Event Sourcing}

The main disadvantage of event sourcing is that it is not suitable for read operations. In event sourcing, the read operation tends to be less responsive than conventional storage methods because a current state is restored by sequentially applying all events included in the domain event.

As event sourcing is not a fully developed technology, there are inadequate tools providing specialized functionality. Therefore, existing technology require improvement or new implementations.

\section{V2X EVENT REPRESENTATION (V2X- ER) ARCHITECTURE}

Figure 1 shows V2X event representation framework. The V2X event representation architecture summarizes the process of processing events and modules.

\subsection{V2X Devices}

V2X devices include any device that generates or sends events, including various sensors.

A sensor periodically transmits collected values at specific times or actively detects changes in an external environment. Most sensors are located at specific points to detect changes in an external environment. Active sensors are often used in $\mathrm{V} 2 \mathrm{X}$ environments.

A machine is a device used to collect and transmit a value generated by a specific sensor or to control a surrounding sensor. In a V2X environment, external signals such as cars and traffic lights, can be collected or change the status of certain situations. Therefore, large data is available for transmission compared to the sensor. Furthermore, varying data messages are used alternate to simple data (value) because they include events occurring in the network and the control device.

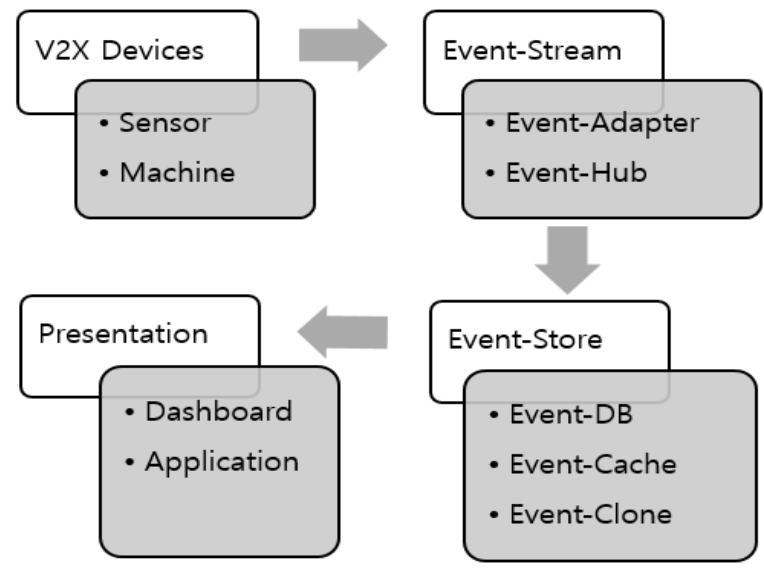

Figure 1. V2X Event Representation Framework.

\subsection{Event-Stream}

Event-Stream is a process of converting a value or message transmitted from a V2X device into an event stream. A stream refers to a virtual passage, and the event stream refers to the process of changing a value or message into an event.

The reason for converting a value or a message into an event stream is preprocessing a process for converting and storing a value or a message generated from various devices into a structure used for event sourcing of a certain type. A value or message stored without transforming into a coherent structure can be expensive for future data verification and status reproduction.

An event-adapter is the main function used to convert a value or message of V2X devices into specific event type. A vital function of an event-adapter is to match an ISO8601 standard with a unique serial number that identifies a value or message generated by a sensor and when it is generated. As a V2X environment collects data from various environments and replays all events of domain events to restore a current state, it is essential to standardize when values or messages are generated. 
An event-hub acts as a temporary storage that can sequentially process values or messages generated by V2X Devices. It provides a function that classifies an event-stream for group or specific purposes by storing a created or converted event in a queue.

A vital role of an event-hub is to temporarily store an event to be processed in an event-store. It utilizes a temporary storage function if the amount of data generated in a V2X environment suddenly increases so that the event-store cannot process it immediately.

It can be processed in event-hub when priority needs to be considered or when the event needs to be sorted and processed.

\subsection{Event-Store}

An event-store provides a function to store an event from an event-stream. The main operations by an event-store are operations that store events, and return state values at specific times. An event-store improves performance through event-cache and event-clone for read operation and data verification, and provides separate function for data verification.

Event-DB(event-database) is mainly used to store events. EventDB provides only write and read operations. Event-DB does not provide an operation to modify or delete an event. To describe a current state, all events related to the time are applied sequentially increasing processing cost for the read function to be processed in an event-DB. The main use of an event-DB is to store events.

Event-cache provides the ability to improve the read performance of stored events and response time. To improve read performance, snapshot [9], a method of precomputing domain events before a given time is provided. Because event sourcing does not allow modifications and deletions, precomputing historical data at a given time can improve read performance and response time.

Event-clone provides the ability to clone stored events. Event-DB does not support modify and delete operations. It provides a function to duplicate an existing data for data verification. Particularly, copying existing data, various methods can be used for data verification. Furthermore, the replication function used as learning data for machine learning and artificial intelligence, provides various data analysis and prediction functions required for V2X.

\subsection{Presentation}

Saved events can be used in varying ways. To process data in a $\mathrm{V} 2 \mathrm{X}$ environment, the data is checked in a time series analysis and the data is processed around a state.

Dashboard is a time series representation of current state values using stored events. These dashboards can reproduce values or status at any time. Expressing an event at a specific time in a time series allows a visual examination of various states of a sensor or device. Data collected in a V2X environment can be reproduced and represented at any time as data at a specific time. Thus, it is possible to reproduce the relationships and the state of various data generated in a V2X environment.

In applications, stored events are used for web or mobile applications. Alternate to representing time series information, these applications are often used to monitor status and present data from a given sensor or device. Therefore, applications to data that does not need to be represented in time series can be considered.

\section{DISCUSSION}

Some difficulties are encountered in implementing event sourcing. A profound difficulty is the diverse structure of events.

Event-store can be used regardless of specific technology, language, and environment if it provides only storage and reading functions. In implementation as a relational database, each event requires one record. A typical complication is that the table structure is not easy to define because event structures vary. In implementation using relational database, there is an advantage of converting events into JSON or XML to serialize and store data. In using document-based NoSQL, no definition of table structure is required making it easier to process events compared to relational database. Using existing repositories requires various studies and discussions on how to apply complex structures used in event sourcing.

To improve read performance and ensure responsiveness, we chose a strategy that condenses existing data and stores it in advance. This strategy is called snapshot [12]. If a repository is specialized for event sourcing, the system judges the process and handles the process. However, if a general relational database or NoSQL is not designed for event sourcing, it must be designed and implemented separately. Various research [13, 14, 15] are being conducted, including snapshots and patterns for processing events such as CQRS (command and query responsibility segregation).

\section{CONCLUSION}

In this study, we proposed an event collection and reproduction architecture for reproducing an accident in autonomous driving. If an accident occurs, the state change that occurs to reproduce the accident and identify the cause is treated as first-class data. Event sourcing is then used to reproduce the state and restore it.

A V2X event representation architecture is suitable for distributed processing based on message delivery. This makes it suitable for managing large data, such as V2X environments. In V2X, various states occurring in the environment can be visually examined to determine a state at a specific time. Data generated by various devices is used to reproduce a specific state when a problem occurred. Furthermore, data validation and analysis are provided so that existing data can be duplicated.

In future studies, we will develop the event collection and reproduction architecture proposed in this paper and examine the proposed model in a real environment via experiments. Additionally, we will study how to process V2X events in real time and models that can predict the state.

\section{ACKNOWLEDGEMENT}

This research was supported by Basic Science Research Program through the National Research Foundation of Korea (NRF) funded by the Ministry of Education (2018R1D1A1B07042967).

\section{REFERENCES}

[1] National Highway Traffic Safety Administration. 2013. Preliminary statement of policy concerning automated vehicles. washington, dc. Verfügbar unter. www.nhtsa.gov/st aticfiles/rulemaking/pdf/Automated_Vehicles_Policy.pdf.

[2] Kiss, G., and Berecz, É. C. 2019. Questions of Security in the World of Autonomous Vehicles, In Proceedings of the 2019 the 5th International Conference on e-Society, e-Learning and e-Technologies. ACM, 109-115. DOI= http://doi.acm. org/10.1145/3312714.3312718. 
[3] Campolo, C., Molinaro, A., Iera, A., Fontes, R. R., \& Rothenberg, C. E. 2018. Towards 5G network slicing for the V2X ecosystem, In 2018 4th IEEE Conference on Network Softwarization and Workshops (NetSoft). IEEE. 400-405. DOI= 10.1109/NETSOFT.2018.8459911

[4] Fowler, M. 2005. Event sourcing 2005. URL: http://martin fowler.com/eaaDev/EventSourcing. html

[5] The Reactive Manifesto. 2014. URL: https://www.reactivem anifesto.org

[6] Erb, B., Meißner, D., Ogger, F., and Kargl, F. 2018. Log Pruning in Distributed Event-sourced Systems. In Proceedings of the 12th ACM International Conference on Distributed and Event-based Systems. ACM. 230-233. DOI= http://doi.acm.org/10.1145/3210284.3219767

[7] Evans, E. 2004. Domain-driven design: tackling complexity in the heart of software. Addison-Wesley Professional.

[8] Meißner, D. 2018. Towards Time Travel in Distributed Event-sourced Systems. In Proceedings of the 12th ACM
International Conference on Distributed and Event-based Systems. ACM, 266-269. DOI= http://doi.acm.org/10.1145/ 3210284.3219499

[9] Fowler, Martin., "Snapshot, 2004." URL: https://martinfowle r.com/eaaDev/Snapshot.html

[10] Meißner, D., Erb, B., and Kargl, F. 2018. Performance Engineering in Distributed Event-sourced Systems. In Proceedings of the 12th ACM International Conference on Distributed and Event-based Systems. ACM, 242-245, 2018. DOI= http://doi.acm.org/10.1145/3210284.3219770

[11] Katsifodimos, A., and Fragkoulis, M. 2019. Operational Stream Processing: Towards Scalable and Consistent EventDriven Applications. In EDBT. 682-685. DOI= 10.5441/002/ edbt.2019.86

[12] Microsoft Docs. 2019. Command and Query Responsibility Segregation (CQRS) pattern 2019. URL: https://docs.micro soft.com/en-us/azure/architecture/patterns/cqrs 